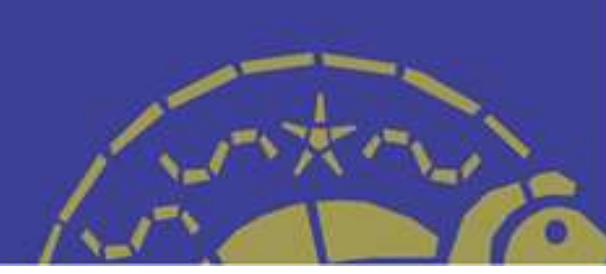

\title{
POPULAÇÃO FEMININA ATENDIDA NO SUS E PREVALÊNCIA DE ALTERAÇÕES CITOLÓGICAS NO COLO DO ÚTERO
}

\author{
Luana Taís Hartmann Backes \\ Universidade de Passo Fundo \\ luana.backes@hotmail.com \\ Adelina Mezzari \\ Universidade Federal do Rio Grande do Sul \\ mezzari@ufrgs.br \\ Luciane Noal Calil \\ Universidade Federal do Rio Grande do Sul \\ Luciane1011@gmail.com
}

\begin{abstract}
Resumo
O presente estudo teve como foco rastrear uma população de mulheres atendidas pelo Sistema Único de Saúde (SUS) e verificar a prevalência, de alterações citológicas no colo do útero. Foi realizada uma busca retrospectiva dos exames SUS, de citologia cervicovaginal realizados no período de janeiro de 2007 a dezembro de 2011 . A coleta dos dados foi realizada a partir dos laudos laboratoriais. No período do estudo foram realizados 62280 exames do SUS, com 2049 (3,3\%) de laudos positivos para algum tipo de lesão, sendo observada uma alta prevalência 36,99\% de alterações citológicas no colo do útero. Este índice é preocupante, o que permite concluir sobre a necessidade de estabelecer uma política de prevenção à saúde de todas as mulheres no nosso país. Este rastreamento serviu de base para discussão de estratégias sobre prevenção, tratamento e controle das lesões precursoras do câncer do colo do útero num projeto de extensão.
\end{abstract}

Palavras-chave: Neoplasias do colo do útero. Papanicolaou. Políticas públicas de saúde. Prevenção.

\section{FEMALE POPULATION ATTENDED THE SUS AND PREVALENCE OF CYTOLOGICAL CHANGES IN CERVICAL}

Abstract

This study focused on screening a population of women served by the Unified Health System (SUS) and to determine the prevalence, cytologic changes in the cervix. It was performed a retrospective search of SUS examinations, cervicovaginal cytology performed from January 2007 to December 2011. Data collection was conducted from laboratory. During the study period were conducted 62280 examinations of SUS, with $2049(3,3 \%)$ of positive reports for some kind of injury, being observed a high prevalence $36,99 \%$ of cytologic changes in the cervix. This index is worrying, which indicates the need to establish a prevention policy to health in all women our country. This screening was the basis for discussing strategies on prevention, treatment and control of precursor lesions of cervical cancer in the extension project.

Keywords: Cervical neoplasia. Papanicolaou. Public health policies. Prevention.

\section{POBLACIÓN FEMENINA ASISTIÓ EN CITOLOGICO Y CAMBIOS EN SUS PREVALENCIA EN CUELLO UTERINO}

Resumen

Este estudio se centró en el cribado de una población de mujeres atendidas por el Sistema Único de Salud (SUS) y para determinar la prevalencia, los cambios citológicos en el cuello uterino. Se realizó una búsqueda retrospectiva de los exámenes SUS, la citología cervicovaginal realizado entre enero de 2007 diciembre de 2011. La recolección de datos se llevó a cabo a partir de los informes de laboratorio. Durante el período de estudio se realizaron 62280 exámenes del SUS, con 2049 (3,3\%) de los informes positivos para algún tipo de lesión, observándose una alta prevalencia 36,99\% de los cambios citológicos en el cuello uterino. Este índice es preocupante, lo que indica la necesidad de establecer una política de 


\section{EXTENEHI

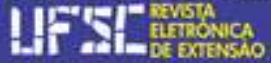

prevención para la salud de todas las mujeres de nuestro país. Esta evaluación fue la base para la discusión de las estrategias sobre prevención, tratamiento y control de las lesiones precursoras del cáncer de cuello uterino en uno proyecto de ampliación.

Palabras clave: Neoplasias cervicales. Papanicolaou. Las políticas de salud pública. Prevention. 
População feminina atendida no SUS e prevalência de alterações citológicas no colo do útero

\section{INTRODUÇÃO}

Atualmente as mulheres são as maiores usuárias dos serviços de saúde, seja privado ou público, em qualquer parte do mundo. Um dos fatores é a conscientização da necessidade em realizar o controle preventivo do câncer através do diagnóstico das lesões precursoras no colo do útero e de mama, cuidados médicos durante a gestação, partos e puerpério, bem como, ao uso de contraceptivos entre outros procedimentos (RIBEIRO et al., 2013).

A implantação do Programa Nacional de Controle do Câncer do Colo do Útero, intitulado "Viva Mulher", criado em 1996, foi definido como prioritário na Política Nacional de Atenção Oncológica e no Pacto pela Saúde, constituindo desta forma um avanço no controle do câncer ginecológico. Antes disso, a realização do exame cito patológico no Brasil, ocorria fora do contexto de um programa organizado, não havendo qualquer mecanismo que estimulasse a procura e garantisse o acesso das mulheres aos serviços de saúde, especialmente aquelas que se encontravam sob maior risco de desenvolver o câncer do colo do útero. Outros programas já existentes apresentavam falhas e seu impacto não refletia a realidade quanto a mortalidade das mulheres com este tipo de câncer; pela falta de organização e continuidade, a não garantia de tratamento adequado nos casos diagnosticados, tampouco o acompanhamento das várias etapas de evolução e seus resultados finais. Por estes motivos, foi implantado o programa "Viva Mulher" estruturado com métodos e medidas contínuas capazes de controlar a doença (SANTOS e GICO, 2005).

Atualmente, as neoplasias representam um problema de saúde pública para todo o mundo, onde o câncer no colo do útero corresponde a aproximadamente $10 \%$ das neoplasias malignas diagnosticadas na população feminina, sendo a segunda causa de morte nas mulheres, superada apenas pelo câncer de mama (TEIXEIRA et al., 2012; MANFREDINI et al., 2013).

Com a implementação de programas organizados de rastreamento, nos países desenvolvidos, uma expressiva redução na morbimortalidade pela doença foi alcançada. Desta forma tanto a incidência como a mortalidade por câncer do colo do útero podem ser reduzidas (SOARES et al., 2011; BORGES et al., 2012).

A incidência deste tipo de câncer tem aumentado a cada ano, sendo que $80 \%$ dos novos casos ocorrem nos países em desenvolvimento, constituindo, portanto, um grave problema a ser enfrentado através das políticas públicas de saúde (BORGES et al., 2012; NOALE et al., 2012; KATKI et al., 2013). Para o ano de 2014 estima-se que ocorram cerca de 15.590 novos casos de 
câncer no colo do útero no Brasil, correspondendo a uma taxa de 15 casos por 100 mil mulheres, sendo que, este mesmo dado equivale aos dados do Rio Grande do Sul (INCA).

Por ser uma doença cuja evolução é lenta e previsível, o câncer de colo uterino apresenta fases pré-invasivas caracterizadas pelas neoplasias intraepiteliais cervicais (NICs). As lesões precursoras são facilmente detectáveis pela citologia preventiva (CARVALHO et al., 2006), oferecida pelo sistema público de saúde. Esta prevenção é realizada através do exame de Papanicolaou, método simples, de baixo custo, o qual faz parte do rol de procedimentos primários de atenção à saúde feminina. Através de coleta de material cervicovaginal na região escamo-colunar, o exame preventivo pode detectar alterações morfológicas na fase pré-maligna, e que ainda são curáveis com a aplicação de medidas relativamente simples. Este controle pode reduzir o risco cumulativo de câncer do colo do útero em cerca de $84 \%$ das mulheres rastreadas a cada cinco anos e em 91\% para mulheres que se submetem ao exame preventivo a cada três anos (GASPERIN et al., 2011; TÜRKMEN et al., 2013).

As alterações morfológicas cervicovaginais são classificadas segundo o Sistema Bethesda 2001 em Atipias de Células Escamosas de Significado Indeterminado (ASC-US), Atipias de células Escamosas as quais não exclui o alto grau (ASC-H), Lesão escamosa intra-epitelial de Baixo Grau (LSIL) se relacionam com a infecção pelo Papilomavírus humano (HPV) e Lesão escamosa intra-epitelial de Alto Grau (HSIL) (SOLOMON e NAYAR, 2005). Na prática, porém, casos limítrofes podem ocorrer, gerando dúvidas entre subestimar ou superestimar um diagnóstico (INCE et al., 2011; TÜRKMEN et al., 2013). A American Cancer Society (ACS) recomenda que o rastreamento preventivo do câncer no colo do útero inicie cerca de três anos após o início da primeira relação sexual, ou antes, dos 21 anos de idade, sendo feito anualmente por meio da citologia, e que seja oferecido pelos sistemas de saúde públicos. Neste caso o índice previsto de casos positivos não deve ultrapassar a 3,5\% (NADPARA et al., 2012).

$\mathrm{Na}$ região norte do Rio Grande do Sul (RS), região do alto Uruguai Gaúcho, pouco se conhece sobre os alcances dos programas de saúde pública focados no rastreamento do câncer de colo uterino, justificando a necessidade de analisar a prevalência das alterações mais frequentes na população desta região. Indo ao encontro deste fato, o objetivo deste estudo foi rastrear uma população de mulheres atendidas pelo Sistema Único de Saúde (SUS) na região do Alto Uruguai, no estado do Rio Grande do Sul (RS) cujo exame citológico foi realizado em um laboratório que atende particulares, convênios e referência no atendimento SUS e verificar a prevalência, nestas mulheres, de alterações citológicas no colo do útero. Este rastreamento foi feito como parte da atividade acadêmica com alunos de graduação com o propósito de avaliar os resultados para 
População feminina atendida no SUS e prevalência de alterações citológicas no colo do útero

servirem de base na discussão das estratégias de orientações sobre prevenção, tratamento e controle das lesões precursoras do câncer do colo do útero e incorporadas num projeto de extensão.

\section{MATERIAIS E MÉTODOS}

Trata-se de um estudo transversal, retrospectivo, que a partir do início do ano de 2012, foi realizado uma busca retrospectiva de todos os exames SUS, de citologia cervicovaginal realizados no período de janeiro de 2007 a dezembro de 2011, num laboratório que atende particulares, convênios e é referência no SUS, localizado na cidade de Erexim, região do Alto Uruguai gaúcho.

Este laboratório é referência na análise de exames preventivos, porém neste estudo foram computados somente os exames SUS, resultando em 62.280 laudos de exames preventivos para o câncer de colo uterino colhidos de pacientes que foram atendidas em 84 Unidades Básicas de Saúde (UBS). A coleta dos dados foi realizada a partir dos laudos laboratoriais mantidos no sistema de resultados do Laboratório. Os critérios de inclusão foram estabelecidos pelos laudos com características pré-malignas (atipias) e de malignidade, e os de exclusão os sem nenhuma alteração.

Foram selecionados somente os laudos citológicos que continham algum tipo de alteração citológica cervicovaginal indicando risco de neoplasia, o que totalizou 2049 resultados. Os critérios de inclusão foram estabelecidos pelos laudos com características pré-malignas (atipias) e de malignidade sendo que os de exclusão os laudos sem nenhuma alteração (SOLOMON e NAYAR, 2005). Nas pacientes com diagnóstico de atipias, houve o acompanhamento pelas UBS, através de contato direto após seis meses do resultado para a realização de um segundo exame, com o intuito de verificar uma possível evolução do quadro e, nos casos com evolução positiva, foram providenciadas as devidas condutas clínicas. A computação dos laudos e o acompanhamento foram retirados do sistema de laudos do Laboratório. Os dados obtidos foram analisados através de testes estatísticos SPSS e analisados por percentuais.

Este estudo foi analisado e aprovado pelo Comitê de Ética em Pesquisa da Universidade Regional Integrada do Alto Uruguai e das Missões URI - Campus de Erechim, reconhecido pelo Comitê de Ética em Pesquisa (CONEP), sob o protocolo número 053/PGH/11. 
População feminina atendida no SUS e prevalência de alterações citológicas no colo do útero

\section{RESULTADOS}

No período do presente estudo, janeiro de 2007 a dezembro de 2011, foram realizados, no referido laboratório, 62280 exames preventivos para o câncer de colo uterino, colhidos de pacientes que foram atendidas no SUS em 84 Unidades Básicas de Saúde (UBS). Destes 2049 (3,3\%) laudos foram positivos para algum tipo de lesão (IC95\%=3,1-3,4\%) e incluídos no presente estudo. Dos resultados positivos, 758 (36,99\%) foram casos de atipias (ASC) e após o contato direto com as pacientes para realizarem o segundo exame, seis meses depois da realização do primeiro, foi observado que destes, $360(47,49 \%)$ - (IC95\%=43,9-51\%) permaneceram com lesões benignas (ASC-US); 225 (29,68\%) - (IC95\%=26,4-32,9\%) evoluíram para lesão de alto grau (ASC-H) e em 173 (22,82\%) - (IC95\%=19,8-25,8\%) casos, as pacientes não responderam ao contato direto das UBS, para a repetição do exame.

Todos os resultados dos exames, do controle do contato direto com as pacientes para a realização do exame controle após seis meses da realização do primeiro e do não retorno das pacientes ao chamado, foram compilados nos laudos do sistema de resultados do referido laboratório. Outros dados como a condição sócio econômica das pacientes não foi alvo do presente estudo, apenas foi enfocado o atendimento no SUS em Unidades Básicas de Saúde.

A idade das pacientes incluídas no presente estudo foi estratificada segundo a Tabela 1. Um fator limitante neste estudo foi no momento da coleta dos dados no sistema de laudos do laboratório, porque não foi previsto a quantificação do número de pacientes relacionadas com a malignidade e a faixa etária.

Tabela 1: Distribuição da faixa etária de acordo com o tipo de lesão.

\begin{tabular}{|l|l|}
\hline \multicolumn{1}{|c|}{ Tipo de alteração citopatológica } & Faixa Etária \\
\hline Atipias (ASC-US, ASC-H ou AGC) & 17 a 60 anos \\
\hline LSIL & 17 a 55 anos \\
\hline HSIL & 21 a 55 anos \\
\hline Carcinoma Epidermóide & 35 a 65 anos \\
\hline Adenocarcinoma & 35 a 65 anos \\
\hline
\end{tabular}

Legendas: ASC-US: Atipias escamosas de significado indeterminado; ASC-H: Atipias escamosas que não pode excluir lesão de alto grau; AGC: Atipias de células glandulares; LSIL: Lesão de baixo grau; HSIL: Lesão de alto grau. 


\section{DISCUSSÃO E CONCLUSÃO}

As ações preventivas e as assistenciais têm impactos diferentes quando nos deslocamos para o campo das políticas de saúde. As atividades assistenciais respondem às necessidades de saúde dos usuários do SUS, e as ações preventivas são planejadas com o intuito de modificar o quadro social da doença e, com isso, no futuro, podem alterar a demanda por serviços essenciais (SOARES et al., 2010). A população atendida neste estudo foram somente as mulheres usuárias do SUS.

Nos países desenvolvidos com programas de prevenção tem sido observada uma significativa diminuição na ocorrência de novos casos de câncer. Este fato ocorre pelo acompanhamento cuidadoso da saúde das mulheres na detecção precoce, permitindo atingir altos percentuais de remissão e cura. Nos países menos desenvolvidos, onde as desigualdades sociais favorecem o controle inadequado da prevenção do câncer uterino os resultados positivos ainda são precários e o Brasil ainda pertence a este grupo (VILLA e PEREIRA, 2009; DIAS et al., 2010). No presente estudo, $29,68 \%$ das mulheres com resultado de atipia evoluíram para casos malignos de câncer e 22,82\% das mulheres não atenderam ao chamado para nova coleta, cuja finalidade era de uma possível evolução da lesão inicialmente benigna, ou mesmo, confirmar a regressão do quadro citológico.

Do ponto de vista de saúde pública, sabe-se que a efetividade do programa de controle do câncer cervicovaginal depende da cobertura populacional alcançada, sendo preconizado que no mínimo $90 \%$ das mulheres devem ser submetidas ao exame citopatológico anualmente (RUSSOMANO et al., 2010; GONÇALVES et al., 2011; BARBOSA et al., 2012; TIDY et al., 2013; LIAO et al., 2013. No presente estudo foi observado que a partir de 2007 até 2011, o número de exames citológicos para a pesquisa de câncer no colo do útero vem aumentado a cada ano, no entanto, não existe o controle de todas as mulheres da região estudada para saber se todas as outras procuram atendimento privado e não o público e também foi verificado que nem todas atenderam ao pedido de retorno para o controle da evolução ou não para malignidade.

Os índices estatísticos propostos pelas diretrizes de citopatologia equivalem a 3,5\% dos resultados de atipias e malignidade. Neste estudo, encontramos uma prevalência de 3,3\% de casos com alterações na citologia cervicovaginal no total dos exames realizados. 
Assim, é importante ressaltar que estas alterações devem ser devidamente acompanhadas através do seguimento, com repetição do exame após seis meses. Isto porque este resultado inicial pode corresponder a um diagnóstico falso negativo no primeiro momento (MANFREDINI et al., 2013), confirmado em estudos que relatam a prevalência de HSIL e câncer escamoso em mulheres com citologia prévia de ASC-US e de ASC-H (DIAS et al., 2010; BARROS et al., 2012. Também, neste estudo, 29,68\% das mulheres diagnosticadas com atipia evoluíram para lesão maligna, quando feito o controle após seis meses do primeiro exame citológico.

Com relação à faixa etária, Tabela 1, foi verificado neste estudo que as lesões precursoras do câncer cervical ocorreram entre os 17 e 55 anos para os casos de atipias (ASC-US, ASC-H e AGC), LSIL e HSIL e entre os 35 a 65 anos para os casos de Carcinoma Epidermóide e Adenocarcinoma. Estes dados permitem afirmar que as mulheres mais jovens apresentaram maior prevalência nos casos de atipias, LSIL e HSIL, ou seja de lesões precursoras enquanto que o carcinoma escamoso e adenocarcinoma foram mais incidentes em mulheres com idade mais avançada, acreditando ser pelo maior tempo de evolução das lesões. Em estudos semelhantes (SOARES et al., 2010; LEITE et al., 2014) a faixa etária das mulheres e o tipo de atipias e de câncer foram também compatíveis com o do presente estudo, mesmo não tendo sido quantificado o número de pacientes relacionadas com a malignidade e a faixa etária.

Os programas de rastreamento sistemáticos da população feminina por meio do exame citopatológico do colo uterino tem sido uma das estratégias públicas mais efetivas, seguras e de baixo custo para detecção precoce do câncer de colo de útero.

Caracterizando as alterações celulares encontradas dentro deste percentual de exames citopatológicos da população feminina da região do Alto Uruguai Gaúcho, concluímos que no acompanhamento das pacientes com diagnóstico de atipias através da repetição do exame em seis meses, demonstrou que $29,68 \%$ das mulheres que inicialmente apresentaram atipia evoluíram para lesão maligna. Este fato reforça a necessidade de manter a vigilância com o acompanhamento permanente das mulheres em idade suscetível de adquirirem câncer do colo do útero, pois se sabe que dependendo do estilo de vida e da exposição aos fatores de risco, a evolução para o câncer pode ocorrer.

Em contrapartida, pacientes que deveriam ter realizado o controle através de um segundo exame após seis meses do primeiro, não o realizaram, mesmo tendo sido feito um contato direto para a realização do mesmo. Este índice é preocupante, principalmente quando não existe interesse ou um controle permanente, o que permite concluir sobre a necessidade de estabelecer 
População feminina atendida no SUS e prevalência de alterações citológicas no colo do útero

uma política de prevenção mais eficaz à saúde destas e de todas as mulheres no nosso país. Os resultados obtidos neste levantamento serviram de base para discussão das estratégias de orientações sobre prevenção, tratamento e controle das lesões precursoras do câncer do colo do útero as quais foram incorporadas a um projeto de extensão.

\section{REFERÊNCIAS}

BARBOSA, G. M. S.; MENDES, S. Q.; SILVA, R. M. A.; TEIXEIRA, T. F. F.; RAFAEL, R. M. R.; BARBOSA, D. P. Epidemiological features of papillomatosis caused by the Human Papillomavirus in women attending the Basic Healthcare Clinic in Nova Iguaçu Country, State of Rio de Janeiro. DST - Jornal Brasileiro de Doenças Sexualmente Transmissíveis, v. 24, n. 4, p. 255-259, 2012.

BARROS, N. K. S.; COSTA, M. C.; ALVES, R. R. F.; VILLA, L. L.; DERCHAIN, S. F. M.; ZEFERINO, L. C.; et al. Association of HPV and Chlamydia trachomatis seropositivity in cases of cervical neoplasia in Midwest Brazil. Journal Medical Virology, v. 84, n. 7, p. 1143 1150, 2012.

BORGES, A. M. S.; FERREIRA, C. H. J.; ALMEIDA, A. M.; GOMES, F. A. Childbirth experience according to a group of Brazilian primiparas. Nurs Plus Open, V. 28, N. 6, P. 844-849, 2012.

BORGES, M. F. S. O; DOTTO, L. M. G.; KOIFMAN, R. J.; CUNHA, M. A.; MUNIZ, P. T. Prevalência do exame preventivo de câncer do colo do útero em Rio Branco, Acre, Brasil, e fatores associados à não realização do exame. Caderno de Saúde Pública, v. 28, n. 6, p. 1156-1160, 2012.

CARVALHO, R.; LEITE, C. G.; PEREIRA, D. R. S.; SOUZA, E. R.; SILVA, S. S. L. Carcinoma de células microinvasivo - relato de caso. Revista Para Medica, v. 20, n. 3, p. 6569, 2006.

DIAS, M. B. K.; TOMAZELLI, J. G.; ASSIS, M. Rastreamento do câncer de colo do útero no Brasil: análise de dados do Siscolo no período de 2002 a 2006. Epidemiologia e Serviços de Saúde, v. 19, n. 3, p. 293-306, 2010.

GASPERIN, S. I.; BOING, A. F.; KUPEK, E. Cobertura e fatores associados à realização do exame de detecção do câncer de colo de útero em área urbana no Sul do Brasil: estudo de base populacional. Caderno de Saúde Pública, v. 27, n. 7, p. 1312-1322, 2011.

GONÇALVES, C. V.; DUARTE, G.; COSTA, J. S. D.; QUINTANA, S. M.; MARCOLIN, A. C. Perdas de oportunidades na prevenção do câncer de colo uterino durante o pré-natal. Ciência \& Saúde Coletiva, v. 16, n. 5, p. 2501-2510, 2011. 
INCA - Instituto Nacional de Câncer. [cerca de 10p.]. Disponível em: http://www.inca.gov.br/ . Acesso em 23 de novembro de 2014.

INCE, U.; AYDIN, O.; PEKER, O. Clinical Importance of "low-grade squamous intraepitehelial lesion, cannot exclude high-grade squamous intraepitehelial lesion (LSIL-H)" terminology for cervical smears. 5- Years analysis of the positive predictive value of LSIL-H compared with ASC-H, LSIL, and HSIL the detectionof high-grade cervical lesions with a review of the literature. Journal Ginecology and Obstetric, v. 121, n. 1, p. 152-156, 2011.

KATKI, H. A.; SCHIFFMAN, M.; CASTLE, P. E.; FETTERMAN, B.; POITRAS, N. E.; LOREY, T.; et al. Five-year risk of recurrence after treatment of CIN 2, CIN 3, or AIS: performance of HPV and Pap cotesting in posttreatment management. Journal Low Genital Tract Disease, v. 17, n. 5, p. 78-84, 2013.

LEITE, M. F.; DE VITTA, F. C. F.; CARNAZ, L.; DE CONTI, M. H. S.; MARTA, S. N.; GATTI, M. A. N.; et al. Knowledge and practice of women regarding cervical cancer in a primary health care unit. Journal of Human Growth and Development, v. 24, n. 2, p. 208-213, 2014.

LIAO, S. Y.; RODGERS, W. H.; KAUDERER, J.; DARCY, K. M.; CARTER, R.; SUSUMU, N.; et al. Endocervical glandular neoplasia associated with lobular endocervical glandular hyperplasia is HPV-independent and correlates with carbonic anhydrase-IX expression: a Gynaecological Oncology Group Study. Brasilian Journal of Cancer, v. 108, n. 3, p. 613-620, 2013.

MANFREDINI, V.; CALIL, L. N.; BACKES, L. T. H. Guia Prático de Citologia Cervicovaginal: texto e atlas. São Paulo: Livrobits; 2013.

NADPARA, P. A.; MADHAYAN, S.; KHANNA, R.; SMITH, M.; MILLER, L. A. Patterns of cervical cancer screening, diagnosis, and follow-up treatment in a state Medicaid fee-forservice population. Population Health Management, v. 15, n. 6, p. 362-371, 2012.

NOALE, M.; LIMONGI, F.; SCAFATO, E.; MSGGI, S.; CREPALDI, G. Longevity and health expectancy in an ageing society : implications for public health in Italy. Ann do Instituto Super Sanità, v. 48, n. 3, p. 292-299, 2012.

OLIVEIRA, Z. M.; SOUZA, R. B.; MALHADO, C. H. M.; ARAÚJO, R. T.; DIAS, J. A. A.; OLIVEIRA, J. S. Revista Saúde e Comunidade, v. 9, n. 3, p. 22-24, 2013.

RIBEIRO, L.; BASTOS, R. R.; RIBEIRO, L. C.; VIEIRA, M. T.; LEITE, I. C. G.; TEIXEIRA, M. T. B. Não adesão às diretrizes para rastreamento do câncer do colo do útero entre mulheres que frequentaram o pré-natal. Revista Brasileira de Ginecologia e Obstetricia, v. 35, n. 7, p. 323-30, 2013. 
RUSSOMANO, F.; MONTEIRO, A. C. S.; MOUSINHO, R. O. O diagnóstico citológico das células escamosas atípicas - uma avaliação crítica das recomendações diagnósticas. Revista Brasileira de Ginecologia e Obstetrícia, v. 30, n. 11, p. 362-373, 2010.

SANTOS, J.; GICO, V. V. Câncer do colo do útero e a política de prevenção. IX Encontro Latino Americano de Iniciação Científica e V Encontro Latino Americano de PósGraduação - Universidade do Vale do Paraíba [cerca de 4p.]. Disponível em: http://www.inicepg.univap.br/cd/INIC 2005/epg/EPG4/EPG4-50\%20. Acesso em $20 \mathrm{de}$ julho 2015.

SOARES, M. C. ; MISHIMA, S. M. ; MEINCKE, S. M. K. ; SIMINO, G. P. R. Câncer de colo uterino: Características das mulheres em um município do sul do Brasil. Revista de Enfermagem, v. 14, n. 1, p. 90-96, 2010.

SOARES, M. C.; MISHIMA, S. M.; SILVA, R. C.; RIBEIRO, C. V.; MEINCKE, S. M. K.; CORREAA, A. C. L. Câncer de Colo Uterino: atenção integral à mulher nos serviços de saúde. Revista Gaúcha de Enfermagem, v. 32, n. 3, p. 502-508, 2011.

SOLOMON, D.; NAYAR, R. Sistema Bethesda para Citopatologia Cervicovaginal. Definição, Critérios e Notas Explicativas. 2a Edição. Rio de Janeiro: Revinter; 2005.

TEIXEIRA, R. A.; VALENTE, J. G.; FRANÇA, E. B. Mortalidade por câncer de colo do útero no Estado de Minas Gerais, Brasil, 2004-2006: análise da magnitude e diferenciais regionais de óbitos corrigidos. Epidemiologia a Serviço da Saúde, v. 21, n. 4, p.549-559, 2012. TIDY, J. A.; BROWN, B. H.; HEALEY, T. J.; DAAYANA, S.; MARTIN, M.; PRENDIVILLE, W.; et al. Accuracy of detection of high-grade cervical intraepithelial neoplasia using electrical impedance spectroscopy with colposcopy. Brasilian Journal of Ginecology and Obstetric, v. 120, n. 4, p. 400-410, 2013.

TÜRKMEN, I. C.; BASSÜLLÜ, N.; ORKMAZ, P. K.; GUNENC, B.; BAYKAL, C. M.; GUDUCÜ, N.; et al. Patients withepithelial cell abnormality in PAP smears: correlation of results with follow upsmears and cervical biopsies. Turk Patoloji Derg, v. 29, n. 3, p. 179184, 2013.

VILLA, M. C. E.; PEREIRA, W. R. As políticas públicas e a atenção ao câncer do colo do útero no Estado de Mato Grosso - uma abordagem crítica. Revista Eletrônica de Enfermagem, v. 11, n. 4, p. 1037-1042, 2009. Disponível em: http://www.fen.ufg.br/revista/v11/n4/pdf/v11n4a31. 
População feminina atendida no SUS e prevalência de alterações citológicas no colo do útero 